\title{
Intravitreal dexamethasone implant as an alternative to systemic steroids as prophylaxis for uveitic cataract surgery: a randomized trial
}

\author{
Aditya Sudhalkar ${ }^{1} \cdot$ Abhay Vasavada $^{1} \cdot$ Deepak Bhojwani $^{1} \cdot$ Viraj Vasavada $^{1} \cdot$ Shail Vasavada $^{1} \cdot$ Vaishali Vasavada $^{1}$. \\ Samaresh Srivastava ${ }^{1}$
}

Received: 2 July 2018 / Revised: 19 June 2019 / Accepted: 1 July 2019 / Published online: 18 July 2019

(c) The Author(s), under exclusive licence to The Royal College of Ophthalmologists 2019

\begin{abstract}
Purpose To determine the utility of the dexamethasone implant (IVD) as an alternative to systemic steroids as prophylaxis against cystoid macular edema (CMO) in patients with chronic, recurrent CMO associated intermediate or posterior uveitis (IU/PU), and cataract undergoing cataract surgery.

Methods This was a randomized, parallel design, and clinical trial. Patients with IU/PU and cataract scheduled for cataract surgery were randomly assigned to receive the IVD concurrently with cataract surgery (Group 1:20 patients) or systemic steroids (Group 2: 23 patients) tapered over 4-6 weeks along with uneventful cataract surgery and routine postoperative care. Patients with glaucoma/contraindications to steroids were excluded. All patients were followed up for 6 months.

Outcome measure Primary-incidence of postoperative CMO. Secondary-the change in BCVA (corrected distance visual acuity) and Central Subfield thickness (CST) and complications. Appropriate statistical analysis was done.

Results The median age was 47.3 \pm 4.23 years (group 1) and $49.12 \pm 5.32$ years (Group 2). One patient (Group 1) and two (Group 2) developed CMO. The BCVA improved significantly in both groups $(p=0.013)$. The CST change was insignificant. Four patients (Group 1) required intraocular pressure (IOP) lowering medications. Three patients (Group 2) required early steroid taper.
\end{abstract}

Conclusions IVD is a good alternative as prophylaxis in IU/PU and cataract in preventing postoperative CMO.

\section{Introduction}

Cataract in chronic, recurrent noninfectious uveitis can be a consequence of the disease process itself or therapy thereof and is an important cause of vision loss in the said condition [1]. Cataract surgery and intraocular lens implantation in

Presented as a free paper at EURETINA in September 2017 in Barcelona, Spain.

This trial has been sponsored in full by Allergan India Pvt Ltd (Bangalore; including provision of study drugs).

All authors contributed equally to the manuscript.

Aditya Sudhalkar

adityasudhalkar@yahoo.com

1 Raghudeep Eye Hospital and Ila Devi Cataract and IOL Research Centre, Ahmedabad, India uveitis remain a technical challenge despite several recent advances in techniques and technology [1-5]. Postoperative inflammation and cystoid macular edema (CMO) remain one of the biggest threats to compromised visual outcomes, albeit temporary in most cases [2-4]. CMO, posterior capsular opacification, epiretinal membranes, and posterior synechiae can all lead to poor visual outcomes, and CMO is one of the most frequently reported complications [1-4]. CMO is particularly common in uveitic cataracts wherein the uveitis has previously been associated with $\mathrm{CMO}$ and can herald recurrence. The incidence of postsurgical CMO is also higher in patients with uveitic cataract $(12-50 \%)$, despite steroid prophylaxis [5-9]. It follows that adequate perioperative control of inflammation in addition to careful surgery and appropriate choice of posterior chamber intraocular lens placement is of paramount importance in such patients [1-9]. Topical, intraocular, periocular, and systemic corticosteroids as well as other immunosuppressants have proven to be successful in controlling inflammation in the postoperative period $[10,11]$. The most 
common therapeutic route used historically is systemic corticosteroids, especially in severe noninfectious intermediate, posterior or panuveitis both as therapy and as prophylaxis $[4,5,10,11]$. The benefit of systemic corticosteroids is somewhat offset by the high incidence of systemic side effects as a consequence of its use [5, 10, 11]. Local steroid therapy has been reported to be useful $[4,5]$ both as prophylaxis [12] and treatment [13].

The intravitreal dexamethasone implant (Ozurdex, Allergan, Irvine, CA) has been shown to be effective and safe in noninfectious recurrent intermediate or posterior uveitis as therapy [14-17]. In addition, there are comparative and noncomparative case series demonstrating its utility as prophylaxis in patients with uveitic cataract undergoing cataract surgery $[18,19]$. The implant is not recommended in pseudophakia complicated by a posterior capsular rent or in aphakes.

We undertook this study to determine whether the intravitreal dexamethasone implant is an alternative to systemic corticosteroids as prophylaxis against $\mathrm{CMO}$ and vision loss in patients with noninfectious recurrent intermediate or posterior uveitis with $\mathrm{CMO}$ and cataract undergoing cataract surgery.

\section{Methods}

This was a randomized, comparative clinical trial conducted at the Raghudeep Eye Hospital, Ahmedabad, India. Ethics Committee approval was obtained prior to initiation of the trial. The trial is registered at www.ctri.nic.in (CTRI/2017/ 08/009444). Informed consent was obtained from the patients prior to enrolment. The study adhered to the tenets of Helsinki. Grant support was received (along with study injections) for the intervention from Allergan India Pvt. Ltd, the marketers and distributors of the intravitreal dexamethasone implant (Ozurdex, Allergan, Irvine,CA). The funders had no role in study design, data collection and analysis, decision to publish, or preparation of the manuscript.

\section{Inclusion and exclusion criteria}

For inclusion, patients were required to meet the following criteria: (1) Age 18 years or older; (2) diagnosis of previous unilateral recurrent noninfectious intermediate uveitis or posterior uveitis with CMO and cataract of sufficient degree to warrant surgery. (3) Well-controlled uveitis for at least 3 months prior to scheduled date of cataract surgery $(<1+$ cells in the anterior chamber and vitreous; SUN classification [20]) without any evidence of CMO at the time of inclusion. However, all patients had had either recurrent intermediate uveitis or posterior uveitis with associated CMO with at least one recurrence in the past.

Patients who were found to have any of the following were excluded from the study: (1) Any systemic disease that is inadequately controlled, according to the best medical judgment and that precludes use of systemic steroids; (2) A known allergy to a required study medication; (3) Uncontrolled/advanced glaucoma or a history of steroid response; (4) Presence of any ocular comorbidity that can influence visual recovery, including complications during cataract surgery such as a posterior capsular rent or vitreous loss; (5) Pregnancy or current breastfeeding; (6) Known human immunodeficiency virus infection or other immunodeficiency disease for which corticosteroid therapy would be contraindicated according to best medical judgment; (7) Medical problems or drug or alcohol dependence problems sufficient to prevent adherence to treatment and study procedures; (8) Past use of triamcinolone acetonide or the fluocinolone implant. The dexamethasone implant was permissible as therapy for uveitis but should not have been used in the study eye in the 6 months prior to inclusion.

\section{Sample size calculation}

Since the study question dealt with prophylaxis against CMO, we chose to formulate a study that detected a $10 \%$ difference in the incidence of CMO between the two groups. A sample size of 18 patients per group was estimated to give $90 \%$ power to detect this difference allowing a type I error probability of 0.05 . Assuming an attrition rate of $10 \%$, it was decided to recruit at least 20 patients in each group. Because one of the groups was to receive systemic therapy and since we included patients with unilateral intermediate or posterior uveitis, patients (and not eyes) were randomized to either group.

\section{Examination, therapy, and follow-up}

All eligible patients underwent a thorough history taking and a comprehensive anterior and posterior segment examination prior to enrolment. They also underwent elaborate investigations to rule out infectious uveitis. All patients received fundus fluorescein angiography prior to surgery along with optical coherence tomography (OCT) raster analysis to rule out the presence of preoperative CMO. Postoperatively, fluorescein angiography was performed only if CMO was detected on OCT, given that visually significant CMO was the focus of interest in this study. Patients were randomized to receive the intravitreal dexamethasone implant at the time of cataract surgery (group 1) or oral corticosteroids beginning 2 days prior to the scheduled date of surgery tapered over 3-4 weeks. A single surgeon (AV) performed cataract surgery 
(phacoemulsification) and IOL implantation. Group 1 patients did not receive any additional topical or systemic immunosuppressive therapy prior to surgery. Group 2 patients did not receive topical immunosuppressive therapy prior to surgery. Postoperatively, all patients received topical prednisolone drops six times a day tapered over 6 weeks along with cycloplegic therapy for a week. Topical therapy also included nepafenac eye drops three times a day for 2 months from postoperative day 1. Topical therapy could be extended in the event of severe inflammation or if CMO was detected at the treating ophthalmologist's discretion. The intravitreal dexamethasone implant was injected using a standardized technique under asepsis by an experienced retinal surgeon (DB) at the time of cataract surgery. If the patient was assigned to the systemic therapy group, prophylactic oral steroids were initiated 2 days prior to the cataract surgery. Oral prednisolone was prescribed at $1 \mathrm{mg} / \mathrm{kg}$ body weight/day. The maximum administered dose was $60 \mathrm{mg} /$ day (dose: $1 \mathrm{mg} / \mathrm{kg}$ body weight). That is to say: patients who weighed more than $60 \mathrm{~kg}$ did not receive a correspondingly higher dose and the maximum that they received on any given day was $60 \mathrm{mg}$, given that these steroids had been prescribed prophylactically and all patients had stable, well-controlled uveitis prior to inclusion in the study. Postoperatively, patients could be given an appropriate dose of systemic steroids in either group if there was significant inflammation. Systemic corticosteroid/ immunosuppressive therapy was always in consult with a physician. Alterations in pre-existing oral/injectable medications for systemic comorbidities (because of the introduction of steroids) was per physician's discretion. Randomization was achieved with the help of a random number table. Additional injections and/or systemic immunosuppression were permissible in the event of uveitis reactivation. Both groups could receive additional local or systemic immunosuppression with the choice of immunosuppressive drug individualized to each patient. Follow-ups were scheduled on days $1,7,15,30,45,60$ and then monthly thereafter up to 6 months postoperatively. The BCVA (corrected distance visual acuity), Goldmann applanation tonometry, slit lamp examination, binocular indirect examination, and +90D slit lamp biomicroscopy were performed at all visits and OCT raster scans (using the Cirrhus OCT equipment by Zeiss India Pvt. Ltd, Bangalore) were obtained using a standardized technique at days 15, 30 and then monthly thereafter. CMO was defined as the appearance of cystic spaces and retinal thickening $>100 \mu \mathrm{m}$ from baseline as noted on spectral domain OCT with or without neurosensory detachment of the fovea (none of the patients had macular edema at the time of inclusion in the trial and at the time of scheduling for cataract surgery). Resolution of CMO was defined as complete disappearance of retinal cysts and subretinal fluid (if present) along with a reduction in retinal thickness to within $50 \mu \mathrm{m}$ of baseline thickness. The development of new symptoms in any patient and/or the detection of recurrent inflammation (SUN criteria) and its sequel could mean more frequent follow-ups at the investigator's discretion.

Intraocular pressure (IOP) was closely monitored, especially in Group 1, with IOP lowering medications instituted if the IOP went beyond $25 \mathrm{~mm} \mathrm{Hg}$ at any visit, based on recommendations [20-22] for ocular hypertension secondary to intravitreal dexamethasone implant usage (glaucomatous damage to the optic nerve was excluded in all the patients who demonstrated raised IOP through objective evaluation). Patients who had an IOP spike greater than $10 \mathrm{~mm} \mathrm{Hg}$ were monitored weekly, but antiglaucoma therapy instituted only if the IOP exceeded $25 \mathrm{~mm} \mathrm{Hg}$.

The surgeon was informed of the group a particular patient was randomized to at the end of an uneventful phacoemulsification and IOL implantation procedure. The primary investigator (AS) and the data analyst were masked to both groups. Masking was applied to data interpretation based on photographs or imaging studies and IOP studies as well as visual acuity changes. The patients were not masked to the therapy being provided. The allocation list was not stored.

\section{Outcome measures}

The primary outcome measure was the difference in incidence of CMO in both groups. The secondary outcome measures were the change in BCVA from baseline in each group at months 1,3 , and 6 as well as a comparison of the change in BCVA between group at months 1, 3, and 6, a comparison of the change in central subfield thickness from baseline as well as between groups at months 1,3 , and 6 and the complications of therapy, including uveitis reactivation, if any in each group.

\section{Statistical analysis}

Descriptive statistics were used to analyze categorical variables in terms of size (absolute frequencies) and percentage (relative frequencies). The Wilcoxon rank sum test was used to compare differences in continuous variables between groups and the Fisher's exact test for analysis of categorical variables, wherever appropriate.

\section{Results}

Between January 2015 and December 2016, a total of 43 patients were enrolled in both groups. Table 1 lists the demographic characteristics of all patients, including the group distribution of uveitic disease and baseline BCVA as 
Table 1 Demographic characteristics of patients in both groups

\begin{tabular}{lcllllc}
\hline Group & Median age (years) & Gender (M/F) & $\begin{array}{l}\text { Baseline CDVA } \\
\text { (logMAR) }\end{array}$ & $\begin{array}{l}\text { Intermediate } \\
\text { uveitis }\end{array}$ & $\begin{array}{l}\text { Posterior uveitis } \\
\text { Median duration of quiescence } \\
(\text { months })^{\mathrm{a}}\end{array}$ \\
\hline $1(n=20)$ & $47.3 \pm 4.23(35-58)$ & $9 / 11$ & $0.48 \pm 0.1 ;(0.24-1.0)$ & 11 & 9 & $9.25 \pm 3.22(4-15)$ \\
$2(n=23)$ & $49.12 \pm 5.32(37-61)$ & $10 / 13$ & $0.54 \pm 0.14(0.3-1.0)$ & 16 & 7 & $11.40 \pm 4(4-19)$ \\
\hline
\end{tabular}

${ }^{\mathrm{a}}$ Median duration of quiescence prior to inclusion in trial and scheduling of cataract surgery

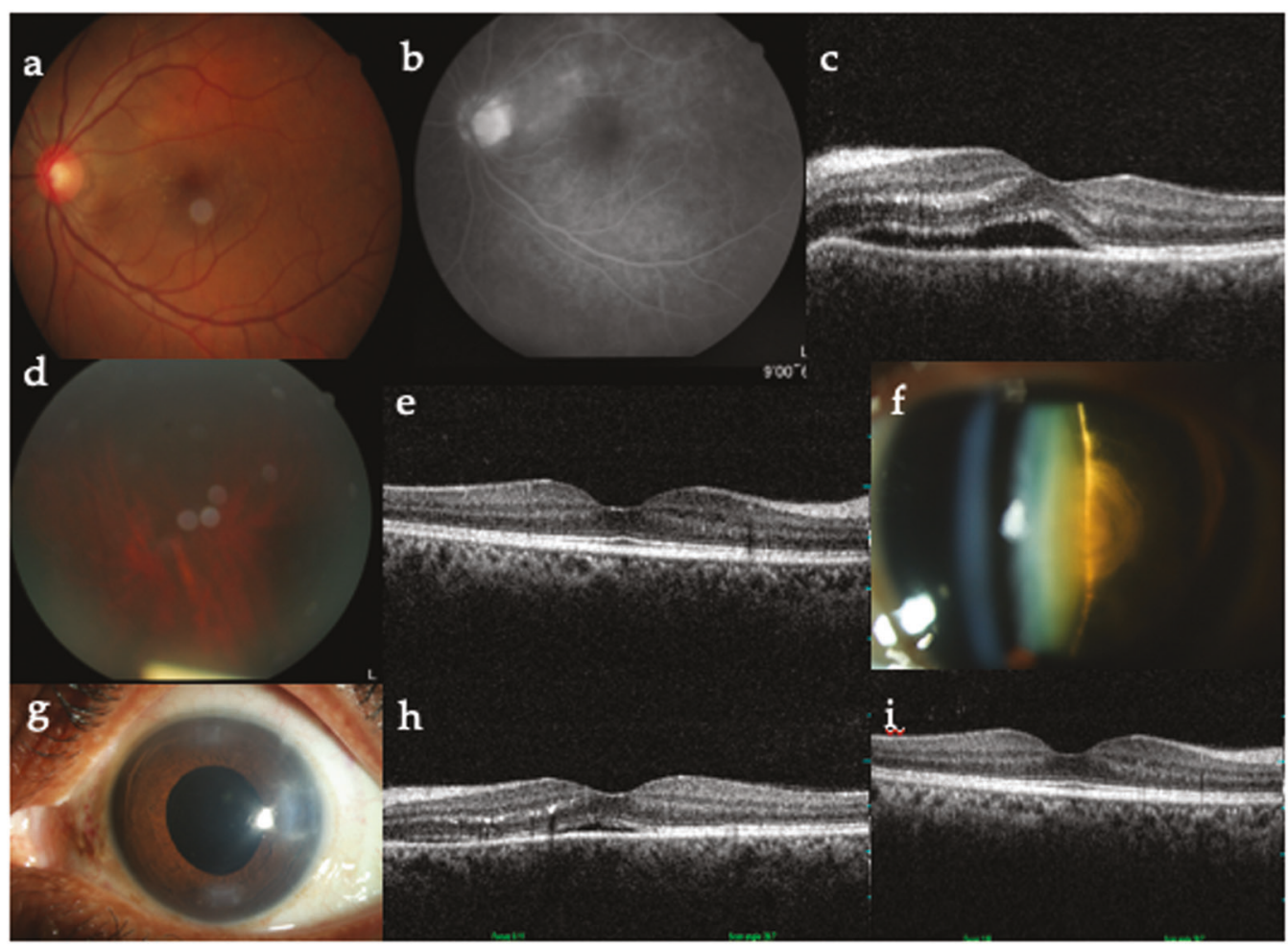

Fig. 1 a Shows the fundus photograph of a 55 years old phakic lady with multifocal choroiditis, confirmed with early hypo fluorescence and late hyper fluorescence (b). The optical coherence tomography (OCT) scan shows retinal thickening with subretinal fluid along with focal hyperintense lesions representing inflammatory aggregates (c). The patient was treated with the intravitreal dexamethasone implant which can be seen in situ (d). The choroiditis resolved completely (e). A year later, the patient developed visually significant cataract and was

well median duration to last episode of inflammation. None of the patients had visually significant cataract in the fellow eye. None of the patients had any known associated systemic disease. A total of nine patients were on maintenance therapy. Four patients in Group 1 and 5 patients in Group 2 were on maintenance therapy with Azathioprine. Three of these were on oral Azathoprine therapy, $50 \mathrm{mg} / \mathrm{day}$. The other six received $100 \mathrm{mg}$ of Azathioprine daily as maintenance therapy. The other patients were not on systemic maintenance therapy at the time of inclusion and none of the patients were on systemic steroids at the time of inclusion. There was no significant difference between groups in terms of age $(p=0.31)$, baseline BCVA $(p=0.42)$ or median duration of quiescence $(p=0.49)$. Four patients in group 1 included in the trial in Group 1 (dexamethasone implant group; f). She underwent uneventful phacoemulsification surgery with in-the-bag IOL implantation (g). She developed recurrent CMO 6 weeks postoperatively as seen on OCT analysis (h) and received a second intravitreal dexamethasone implant injection which led to resolution of CMO (i). She did not demonstrate a recurrence till the end of the follow-up period

and six patients in group 2 had multifocal choroiditis. All other patients in both groups had idiopathic intermediate uveitis. None of the patients in any group had glaucoma or a history of steroid response. None of the patients had had a dexamethasone implant injection within 6 months of cataract surgery. None of the patients had any known contraindication to the use of steroids. Twelve patients had systemic comorbidities (well-controlled medically): Three patients in Group 1 and 2 patients in Group II had type 2 diabetes mellitus, two patients in group 1 and 2 patients in Group II were both hypertensive (primary hypertension) and type 2 diabetics. Three patients (two in Group 1 and one in Group 2) had hypertension and dyslipidemia. Figure 1 depicts an illustrative case. Figure 2 represents 


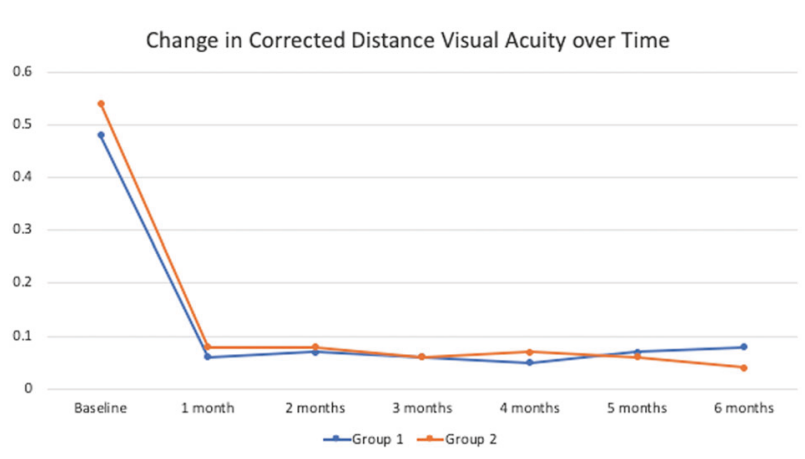

Fig. 2 Demonstrates the change in corrected distance visual acuity over time for both groups. As is evident, both groups showed significant improvement in BCVA postoperatively. There was, however, no significant difference between the two groups in terms of visual acuity till the end of the follow-up period

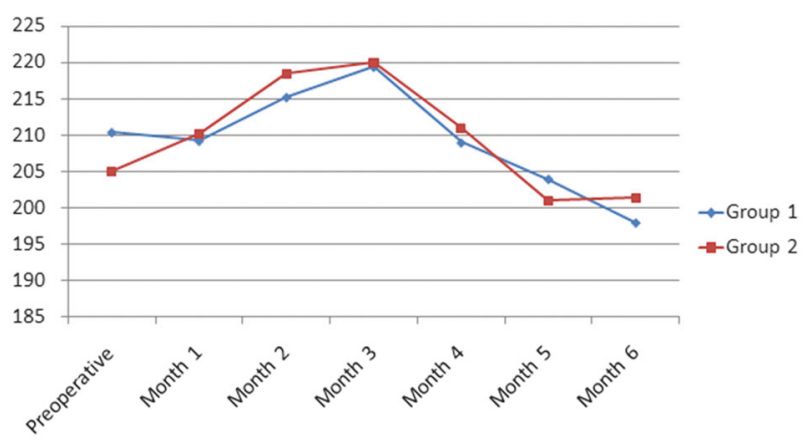

Fig. 3 Demonstrates the change in Central Subfield Thickness (CST) over time for both groups. As evident, there was an insignificant change in CST over time during the follow-up period for both groups overall. The patients who developed postoperative cystoid macular edema in both groups have been described

change in visual acuity over the follow-up period. Figure 3 demonstrates the change in central subfield thickness over time.

The mean time to the last episode of macular edema was 12.20 months (SD-2.46 months, range 8-16 months) in Group 1 and 13.42 months (SD-3.1 months; range 8-15 months) in Group 2.

The median IOP in Group 1 was $14.25 \mathrm{~mm} \mathrm{Hg}$ (SD-3.12 $\mathrm{mm} \mathrm{Hg}$; range $10-19 \mathrm{~mm} \mathrm{Hg}$ ). The median IOP in Group 2 was $16.4 \mathrm{~mm} \mathrm{Hg}$ (SD-4.11 $\mathrm{mm} \mathrm{Hg}$; range 9-19 $\mathrm{mm} \mathrm{Hg}$ ) (Tables 2 and 3). Overall (according to the LOCS III classification), 13 patients had nuclear sclerosis (Grade I-IV), 21 patients had nuclear sclerosis (Grade I-IV) with posterior subcapsular cataract and nine patients had nuclear sclerosis (Grade I-IV) with posterior subcapsular and cortical cataract.

All patients in both groups underwent uneventful phacoemulsification and IOL implantation. One patient in Group 1 developed CMO by month 3 (Fig. 1). She received a second intravitreal dexamethasone injection and did not show recurrence during the rest of the follow-up period. Her postoperative BCVA at month 6 was $0.06 \log$ MAR. Two patients in Group 2 developed CMO in the postoperative period; one developed $\mathrm{CMO}$ a month after complete cessation of steroid therapy. The second developed CMO 3 months after cataract surgery. Both patients were treated with intravitreal dexamethasone implant injections and showed complete resolution of CMO without further recurrences during follow-up. The final postoperative BCVA in both patients was $0.04 \log$ MAR and $0.06 \log$ MAR, respectively. The vision in both groups remained stable and the same during the follow-up period (Fig. 2). The difference between the postoperative and baseline BCVA in each group was statistically significant at all points in time during the follow-up period (Group 1: $p=$ 0.012. Group 2: $p=0.013$ ). However, there was no significant difference between the two groups in terms of visual acuity during the follow-up period as can be seen in Fig. 2 ( $p=0.42$ at 6 months).

Only 4 patients (Group 1) developed ocular hypertension during the follow-up period. One patient demonstrated a rise in IOP at month 1, while the rest had a rise in IOP as noted at the 2 months visit. Three of these patients were managed well with monotherapy for IOP control while one patient required two topical medications. The IOP returned to baseline in all of these four patients by month 6 . None of these patients required incisional surgery for lowering the IOP.

Three patients in Group 2 required early rapid taper of systemic steroids (under the supervision of a physician) because the drug adversely affected their blood glucose control.

There was no significant change in CST postoperatively in either group as considered from baseline, barring the three patients described above (Group 1: $p=0.33$; Group 2: $p=0.45$ ). There was no significant difference in the CST between the two groups during the entire course of follow up (Fig. 3; $p=0.47$ at 6 months).

Except for the three patients described above, none of the patients in Group 1 required systemic immunosuppressive therapy at any point in time during the follow-up period. Conversely, none of the patients in Group 2 required additional systemic immunosuppression and/or local steroid therapy at any point in time during the follow-up period.

No other complications were noted during the follow-up period. Figures 1, 2, and 3 depict the preoperative and postoperative course of a patient with cataract and posterior uveitis who was randomized to group 1 (Steroid implant) and who had an uneventful postoperative course until the end of follow up.

\section{Discussion}

We report good results in this small, randomized trial with the use of the intravitreal dexamethasone implant as 
Table 2 Change in CDVA (corrected distance visual acuity) postoperatively (postop) from baseline at different points in time

\begin{tabular}{llllll}
\hline Groups & Baseline CDVA & 1 month postop & 3 months postop & 6 months postop & $P$ value (intragroup) \\
\hline 1 & $0.48 \pm 0.1 ;(0.24-1.0)$ & $0.06 \pm 0.04(0.0-0.2)$ & $0.06 \pm 0.05(0.0-0.22)$ & $0.08 \pm 0.05(0.02-0.2)$ & 0.012 \\
2 & $0.54 \pm 0.14(0.3-1.0)$ & $0.08 \pm 0.04(0.0-0.24)$ & $0.06 \pm 0.05(0.0-0.22)$ & $0.04 \pm 0.06(0.0-0.024)$ & 0.013 \\
$p$-value (intergroup) & 0.32 & 0.44 & 0.42 & 0.42 & \\
\hline
\end{tabular}

All CDVA values are in $\log$ MAR

Table 3 Changes in Central Subfield Thickness (CST) values from baseline at different points in time

\begin{tabular}{llllll}
\hline Groups & Baseline CST & Month 1 Postop & Month 3 Postop & Month 6 Postop & $p$ value (intragroup) \\
\hline 1 & $205 \pm 17(165-253$ & $210 \pm 14.2(172-247$ & $203.2 \pm 15(171-245)$ & $206.5 \pm 16.4(165-250)$ & 0.33 \\
2 & $210 \pm 14(174-248)$ & $209.4 \pm 16.23(172-249)$ & $202 \pm 18.24(178-252)$ & $198 \pm 14(169-244)$ & 0.45 \\
$p$ value & 0.47 & 0.42 & 0.44 & 0.47 & \\
\hline
\end{tabular}

All CST (Central Subfield thickness) values are in microns

prophylaxis in patients with quiescent intermediate or posterior uveitis and cataract scheduled for cataract surgery. The use of the dexamethasone implant prophylactically in these patients demonstrated immunosuppressive effects which were similar in efficacy to systemic steroids and avoided entirely the systemic side effects of oral steroids. There was no significant difference between the two groups in terms of the incidence of CMO (5\% versus $8 \%$ ), gain in BCVA or change in CMT. A fifth of the patients were found to have developed ocular hypertension in the dexamethasone implant group; however, none of the patients needed incisional surgery for IOP control and the IOP returned to baseline by month 6 . Three patients in Group 2 required early taper of steroids due to uncontrolled blood sugar levels.

The incidence of ocular hypertension in Indian patients with uveitis who receive intravitreal dexamethasone therapy has shown to vary from $7.6 \%$ to $15 \%$ in short series [23-26], a figure comparable to ours. It is also possible that the small sample size in our trial might throw up a disproportionately higher or lower incidence of ocular hypertension.

The intravitreal dexamethasone implant is approved for use in intermediate and posterior uveitis [21] and has shown good efficacy and safety as therapy for the aforementioned conditions. Patients with cataract associated with any form of recurrent uveitis generally receive prophylactic immunosuppressive therapy perioperatively in a bid to reduce the probability of developing postoperative CMO as well reactivation of uveitis, the most short term common complications of cataract surgery in such situations [4-6, 18]. Systemic steroids are by far the most commonly used therapy (with oral steroids the most commonly used route of administration) in this setting $[1,4,5]$. The side effects of systemic therapy with steroids are all too well known and local alternatives to steroid regimens are indubitably safer and perhaps preferable as long as they can produce similar results. Studies on the intravitreal dexamethasone [14-19, 27] and the fluocinolone acetonide [13] implant have demonstrated reasonably well the efficacy and safety of local therapy for active uveitis. The current study is an attempt to demonstrate the utility of the dexamethasone implant as prophylaxis against $\mathrm{CMO}$ and/or recurrent intermediate or posterior uveitis in patients with uveitis associated cataracts.

The utility of triamcinolone acetonide perioperatively $[28,29]$ at the time of cataract surgery in patients with uveitis is known. Patients who received local therapy showed fewer recurrences, improved vision and fewer inflammatory sequelae. Perioperative use of the dexamethasone implant for uveitic patients undergoing cataract surgery has been reported earlier courtesy case reports and noncomparative and comparative case series [18, 19, 27, 30]. Indeed, its successful use in anterior uveitis has also been published [19]. Most case series demonstrate good visual recovery and insignificant changes in CMT with minimal or no instances of complications, particularly IOP increases. The American Academy Preferred Practice Patterns guidelines (2013) [31] state that whereas there is good evidence to show the efficacy and safety of the intravitreal dexamethasone implant as prophylaxis at the time of cataract surgery, there has been no randomized trial conducted thus far to address this question. Since then, as discussed earlier, comparative randomized and nonrandomized case series and case reports have documented the use of the dexamethasone implant as prophylaxis in patient with uveitis undergoing cataract surgery.

The incidence of postoperative CMO is higher in patients with uveitis undergoing cataract surgery as opposed to controls [26]. The incidence of CMO despite prophylaxis ranged from $12 \%$ [9] to as high as 50\% [5-7]. Rahman et al. reported a lower incidence of CMO (8.3\%). However, their series had a significant proportion of patients who had cataract associated with Fuch's heterochromic iridocyclitis. 
Also, their diagnosis of CMO was purely clinical. Conversely, Ram et al. reported no CMO with minimal or no change in CMT as noted on OCT scans [18]. The varying incidence of CMO postoperatively can probably be attributed to the inclusion of patients with differing severity of uveitis as well as to different sites of inflammation (anterior, intermediate or posterior). The long-term outcomes of cataract surgery in patients with uveitis has been discussed before [32, 33].

Small numbers and a short follow up could be considered a limitation of our study. Also, we had stringent inclusion criteria including unilaterality of uveitis and cataract. This obviously led to lower numbers being recruited. However, the study criteria were met. An intact posterior capsule in particular and uneventful cataract surgery in general is a prerequisite for intravitreal dexamethasone implant injection, thereby suggesting that these patients had a good prognosis, visual or otherwise to begin with, especially when one considers that they also had well-controlled uveitis. The low rate of CMO recurrence also probably means that these patients had chronic, recurrent but possibly not severe disease. In addition, the design of this trial meant that patient and to an extent investigator masking was technically difficult and hence not performed. That said, there are points of interest in the study. The study demonstrates that in most cases, concurrent phacoemulsification, IOL implantation, and implant injection are a good alternative to the use of systemic immunosuppression, thereby avoiding entirely the complications of systemic steroid therapy and that it does not necessarily elevate the risk of uveitis recurrence or CMO. Also, the use of the implant prevented vision loss much the same way as it did in the systemic steroids group. None of the patients who developed ocular hypertension during the course of follow up required more than two drugs for adequate control of IOP. None of these patients demonstrated persistent IOP rise during the follow-up period and the IOP returned to baseline by month 6 in all patients. Though the number of patients in each arm was small, the fact that three patients overall were detected to have postoperative CMO probably meant that the study was sufficiently powered to detect the said complications. The short follow up of 6 months is in line with the aim of the trial: to determine whether intravitreal dexamethasone therapy shows an equivalent effect to systemic therapy as prophylaxis against postoperative $\mathrm{CMO} /$ uveitis reactivation in uveitic patients undergoing cataract surgery. Finally, our results correspond very well to a recently published randomized study from India [34]. Gupta et al. demonstrate that the dexamethasone implant is a safe and effective option in controlling postoperative inflammation after cataract surgery.

To conclude, we suggest that the intravitreal dexamethasone implant is a safe and effective alternative to systemic steroids as prophylaxis against $\mathrm{CMO}$ and postoperative inflammation in patients with quiescent intermediate or posterior uveitis who undergo uneventful phacoemulsification cataract surgery along with posterior chamber IOL implantation. Additional injections, when necessary, can be administered safely. This route of steroid administration virtually eliminates all the side effects of systemic therapy.

\section{Summary}

\section{What was known before}

- Prophylaxis against recurrent uveitis for patients with uveitis and cataract undergoing cataract surgery is recommended and generally carried out through the administration of oral steroids, with all their attendant side effects

\section{What this study adds}

- The trial looks at the role of the intravitreal dexamethasone implant as prophylaxis in patients with recurrent intermediate or posterior uveitis and cataract undergoing cataract surgery.

- This trial demonstrates that the implant is noninferior to systemic steroids as prophylaxis and helps eliminate the side effects of systemic steroids.

\section{Compliance with ethical standards}

Conflict of interest The authors declare that they have no conflict of interest.

Publisher's note: Springer Nature remains neutral with regard to jurisdictional claims in published maps and institutional affiliations.

\section{References}

1. Van Gelder R, Leveque T. Cataract surgery in the setting of uveitis. Curr Opin Ophthalmol. 2009;20:42-5.

2. Androudi S, Ahmed M, Fiore T, et al. Combined pars plana vitrectomy and phacoemulsification to restore visual acuity in patients with chronic uveitis. JCRS. 2005;31:472-8.

3. Malinowski S, Pulido J, Folk J. Long-term visual outcome and complications associated with pars planitis. Ophthalmology. 1993;100:818-25.

4. Foster CS, Rashid S. Management of coincident cataract and uveitis. Curr Opin Ophthalmol. 2003;14:1-6.

5. Agrawal R, Murthy S, Ganesh S, et al. Cataract surgery in uveitis. Int J Inflamm. 2012;2012:548453. https://doi.org/10.1155/2012/ 548453. 
6. Ram J, Gupta A, Kumar S, et al. Phacoemulsification with intraocular lens implantation in patients with uveitis. J Cataract Refract Surg. 2010;36:1283-8.

7. Ganesh SK, Babu K, Biswas J. Phacoemulsification with intraocular lens implantation in cases of pars planitis. J Cataract Refract Surg. 2004;30:2072-6.

8. Tomlins PJ, Sivaraj RR, Rauz S, et al. Long-term biocompatibility and visual outcomes of a hydrophilic acrylic intraocular lens in patients with uveitis. J Cataract Refract Surg. 2014; 40:618-25.

9. Belair M-L, Kim SJ, Thorne JE, et al. Incidence of cystoid macular edema after cataract surgery in patients with and without uveitis using optical coherence tomography. Am J Ophthalmol. 2009;148:128-35.e2.

10. Jabs DA, Rosenbaum JT, Foster CS, et al. Guidelines for the use of immunosuppressive drugs in patients with ocular inflammatory disorders: recommendations of an expert panel. Am J Ophthalmol. 2000;130:492-513.

11. Nussenblatt RB, Palestine AG, Chan CC, et al. Standardization of vitreal inflammatory activity in intermediate and posterior uveitis. Ophthalmology. 1985;92:467-71.

12. van Kooij B, Rothova A, de Vries P. The pros and cons of intravitreal triamcinolone injections for uveitis and inflammatory cystoid macular edema. Ocul Immunol Inflamm. 2006;14:73-85.

13. The Multicenter Uveitis Steroid Treatment Trial Research Group. The multicenter uveitis steroid treatment (MUST) trial: rationale, design and baseline characteristics. Am J Ophthalmol. 2010;149:550-61e10.

14. Whitcup SM, Robinson MR. Development of a dexamethasone intravitreal implant for the treatment of noninfectious posterior segment uveitis. Ann N Y Acad Sci. 2015;1358:1-12.

15. Kupperman BD, Blumenkranz MS, Haller JA, et al. Randomized controlled study of an intravitreous dexamethasone drug delivery system in patients with persistent macular edema. Arch Ophthalmol. 2007;125:309-17.

16. Williams GA, Haller JA, Kupperman BD, et al. Dexamethasone posterior-segment drug delivery system in the treatment of macular edema resulting from uveitis or irvine-gass syndrome. Am J Ophthalmol. 2009;147:1048-1054.

17. Palla S, Biswas J, Nagesha CK. Efficacy of Ozurdex implant in treatment of noninfectious intermediate uveitis. Indian J Ophthalmol. 2015;63:767-7.

18. Gupta A, Ram J, Gupta A, Gupta V. Intraoperative dexamethasone implant in uveitis patients with cataract undergoing phacoemulsification. Ocul Immunol Inflamm. 2013;21:462-7.

19. Cordero-Coma M, Garzo I, Calleja S, et al. Preoperative cataract surgery use of an intravitreal dexamethasone implant (Ozurdex) in a patient with juvenile idiopathic arthritis and chronic anterior uveitis. J AAPOS. 2013;17:632-4.
20. Goñi FJ, Stalmans I, Denis P, et al. Elevated intraocular pressure after intravitreal steroid injection in diabetic macular edema: monitoring and management. Ophthalmol Ther. 2016;5:47-61.

21. Haller JA, Bandello F, Belfort R Jr, Blumenkranz MS, Gillies M, Heier $\mathrm{J}$, et al. Randomized, sham-controlled trial of dexamethasone intravitreal implant in patients with macular edema due to retinal vein occlusion. Ophthalmology. 2010;117:1134-.e3.

22. National Collaborating Centre for Acute Care. Glaucoma: diagnosis and management of chronic open angle glaucoma and ocular hypertension. NICE clinical guidelines, no. 85. London: National Collaborating Centre for Acute Care (UK); 2009.

23. Jabs DA, Nussenblatt RB, Rosenbaum JT. Standardization of uveitis nomenclature (SUN) Working Group. Standardization of uveitis nomenclature for reporting clinical data. Results of the first international workshop. Am J Ophthalmol. 2005;140:509-16.

24. Nagpal M, Mehrotra N, Juneja R, Jain H. Dexamethasone implant $(0.7 \mathrm{mg})$ in Indian patients with macular edema: real-life scenario. Taiwan J Ophthalmol. 2018;8:141-8.

25. Palla S, Biswas J, Nagesha CK. Efficacy of Ozurdex implant in treatment of non-infectious intermediate uveitis. Indian J Ophthalmol. 2015;63:767-70.

26. Chabblani J, Bansal P, Veritti D, et al. Dexamethasone implant in diabetic macular edema in real-life situations. Eye (Lond). 2016;30:426-30.

27. Lowder C, Belfort R Jr, Lightman S, et al. Dexamethasone intravitreal implant for noninfectious intermediate or posterior uveitis. Arch Ophthalmol. 2011;129:545-53.

28. Li J, Heinz C, Zurek-Imhoff B, et al. Intraoperative intraocular triamcinolone injection prophylaxis for post-cataract surgery fibrin formation in uveitis associated with juvenile idiopathic arthritis. $\mathbf{J}$ Cataract Refract Surg. 2006;32:1535-9.

29. Alkawas AA, Hamdy AM, Shahien EA. Intraoperative intravitreal injection of triamcinolone acetonide for cataract extraction in patients with uveitis. Ocul Immunol Inflamm. 2010;18:402-7.

30. Larochelle MB, Smith J, Dacey MS. Dexamethasone intravitreal implant in the treatment of uveitic macular edema in the perioperative cataract setting: a case series. Am J Ophthalmol. 2016;166:149-153.

31. American academy of ophthalmology preferred practice patterns: uveitis and cataract surgery. https://www.aao.org/clinical-questions/ uveitis-cataract-surgery. Accessed 4 Oct 2017.

32. Baheti U, Siddique S, Foster S. Cataract surgery in patients with history of uveitis. Saudi J Ophthalmol. 2012;26:55-60.

33. Rahman I, Jones N. Long-term results of cataract extraction with intraocular lens implantation in patients with uveitis. Eye (Lond). 2005;19:191-7.

34. Gupta G, Ram J, Gupta V, Singh R, Bansal R, Chawla-Gupta P, et al. Efficacy of Intravitreal dexamethasone implant in patients of uveitis undergoing cataract surgery. Ocul Immunol Inflamm. 2018;21:1-9. 